ISSN: $1560-9111$

\title{
Análisis en el cálculo de la discrepancia óseo dental de forma manual y en la aplicación I Model Analysis 2
}

\author{
Analysis in calculating Osseo dental discrepancy manually and in I \\ Model Analysis 2 application
}

\begin{abstract}
Resumen
Objetivo: determinar si existe diferencia entre el cálculo de la discrepancia oseodentaria de forma manual y utilizando la aplicación iModelanalisys2. Materiales y método: Se conformó una muestra por conveniencia de 120 modelos de estudio pretratamiento de ortodoncia. Se procedió a realizar la medición de todos los órganos dentarios tanto de la arcada superior como inferior, así como la longitud total de ambos arcos con un calibrador digital y calculadora. Posteriormente se realizó el calculo de la discrepancia oseodental introduciendo los datos obtenidos en la aplicación iModelAnalysis2, en un Smartphone. Resultados: El promedio de edad de la muestra fue de 17 ańos. En el maxilar la media de la discrepancia ósea dentaria al calcularlo de forma manual fue de $-3.7 \mathrm{~mm}$ y al calcularla con el programa iModelAnalysis 2 el promedio de la discrepancia óseo dentaria fue de $-3.22 \mathrm{~mm}$. En la mandíbula, la diferencia entre utilizar la aplicación y hacerlo de forma manual presentó una discrepancia de $0.5 \mathrm{~mm}$. Al realizar la comparación de los resultados mediante pruebas de t de Student, no se encontraron diferencias estadísticamente significativas. Conclusiones: realizar el calculo de la discrepancia osea dentaria en forma manual o utilizando la aplicación para smartphone iModelanalisys 2 no alterará el diagnóstico. Palabras clave: cálculo, discrepancia, oseo dentaria,
\end{abstract}

\begin{abstract}
Objective: Determine whether there is a difference between the calculation of the discrepancy osseodental manually and using the iModelanalisys 2 application. Materials and method: A convenience sample of 120 models pretreatment orthodontic study was formed. We proceeded to perform the measurement of all dental organs of the upper and lower arch, and the total length of both arches with a digital caliper and calculator. Subsequently, the calculation discrepancy oseodental entering the data obtained in the iModelAnalysis 2 application in a smartphone. Results: The average age of the sample was 17 years. In the maxillary average dental bone discrepancy to manually calculate it was $-3.7 \mathrm{~mm}$ and calculate the average program iModelAnalysis 2 dental bone discrepancy was $-3.22 \mathrm{~mm}$. In the mandible, the difference between using the application and do it manually discrepancy of $0.5 \mathrm{~mm}$. When comparing the results using Student's t test, no statistically significant differences were found. Conclusions: Realising the calculation of the osseodental discrepancy manually or using the smartphone application iModelanalisys 2 not alter jthe diagnosis.
\end{abstract}

Key words: calculation, discrepancy, osseodental

\section{Introducción}

Para el diagnóstico y elaboración del plan de tratamiento adecuado en ortodoncia son necesarios diversos recursos como las radiografías panorámica y lateral de cráneo. En conjunto, son necesarios modelos de estudio en yeso de los pacientes con los cuales podremos observar algunos detalles que en ocasiones no se observan clínicamente, por ejemplo: simetría, inclinaciones dentarias, forma, tamaño y posición de los dientes, así como para efectuar diversos análisis ortodóncicos. ${ }^{1}$
Uno de los primeros en realizar investigaciones sobre el tamaño dental fue Black en el siglo XIX y aun en la actualidad sus tablas de promedios siguen usándose como referencia. Bolton continúo con este tipo de estudios comparando la masa dentaria de los dientes superiores e inferiores. ${ }^{2}$ Algunos estudios han descrito la relación entre el dimorfismo sexual, la influencia de la etnia y el tipo de maloclusión en el tamaño de los órganos dentarios. Sin embargo, generalmente estas variables no son consideradas en el diagnóstico. ${ }^{3}$

\section{Santiesteban-Ponciano Fabián Alejandro', Gutiérrez-Rojo María Fernanda', Gutiérrez-Rojo Jaime-Fabián²}

1. Estudiante de la Especialidad de Ortodoncia de la Universidad Autónoma de Nayarit.

2. Docente de la Especialidad de Ortodoncia y de la Unidad Académica de Odontología de la Universidad Autónoma de Nayarit

\section{Correspondencia}

Santiesteban-Ponciano Fabián-Alejandro cd.fasantiestebanp@live.com.mx

Dirección postal: Rio Colorado \#95-G, Los Fresnos, Tepic, Nayarit. C.P. 63197

Teléfono: +52 (664)2939434

\section{Coautores:}

Gutiérrez-Rojo María-Fernanda fernanda_mfgr@hotmail.com

Gutiérrez-Rojo Jaime-Fabián jaime.gutierrez@uan.edu.com

Fecha de recepción: 03/05/ 2016 Fecha de aceptación :22/08/2016

En el diagnóstico en ortodoncia es de gran importancia verificar el tamaño de los dientes y el grado de apińamiento dental, ya que el tratamiento podría variar por la magnitud de este. El apińamiento puede ser resultado de una falta de espacio, por lo cual es necesario comparar el espacio disponible y el espacio que se requiere para poder alinear los dientes de forma correcta. ${ }^{4,5}$

Para realizar el análisis óseo dentario es necesario determinar el espacio disponible y el espacio requerido. El espacio requerido es la suma del ancho mesio- 
distal de todos los dientes anteriores a los primeros molares en ambas arcadas. El espacio disponible se realiza con la medición de la longitud del arco iniciando en mesial del primer molar a mesial del primer molar contralateral, esto puede hacerse de dos formas: al medir el arco dental por segmentos, y la otra forma medir con un alambre sobre la superficie oclusal en los puntos de contacto. ${ }^{6}$

La diferencia entre el espacio disponible y el espacio requerido dará el grado de apińamiento de cada paciente, teniendo un valor negativo sabremos que existe una falta de espacio para el acomodo de los dientes, si el valor es menor de los cuatro milímetros se podría hacer desgastes interproximales y expansión de las bases óseas y si el espacio es mayor de los menos cinco milímetros se pensara en un tratamiento de extracciones dentales o en tratamientos ortodóncico quirúrgicos; Los valores positivos nos darán en consecuencia una reserva de espacio. ${ }^{6,7,8}$

Es muy importante verificar el ancho mesiodistal de los dientes para calcular la discrepancia oseodentaria y poder determinar el grado de apiñamiento. Hoy en día, la mayoría de las persona cuentan con un teléfono inteligente, del cual podremos auxiliarnos utilizando una sencilla aplicación

Tomando en cuenta la tecnología, un smarthphone es un dispositivo que cuenta con funciones avanzadas a diferencia de un teléfono convencional. La aplicación iModelAnalysis2 es una aplicación que permite realizar varios análisis de dentición para el diagnóstico de ortodoncia. Por lo que con esta aplicación podremos realizar el análisis de discrepancia óseo dentaria al introducir el ancho mesiodistal de los dientes del maxilar y de la mandíbula, posteriormente el perímetro de la arcada, y el programa hace la operación matemática, obteniendo el resultado de la discrepancia oseodentaria.

\section{Materiales y método}

La investigación es de tipo descriptivo, no experimental y transversal. Las variables a considerar fueron: espacio disponible maxilar, espacio disponible inferior, espacio requerido superior y espacio requerido inferior. Se conformó una muestra al azar de 120 modelos de estudio pretratamiento de ortodoncia de los archivos de la Especialidad de Ortodoncia de la Universidad Autónoma de Nayarit del periodo 2010 al 2015 .
Se incluyeron todos los modelos con dentición permanente desde primer molar derecho hasta primer molar izquierdo, que fueran hechos en la misma Unidad de Radiología dentomaxilofacial y que tuvieran los órganos dentarios a medir totalmente erupcionados. Se excluyeron modelos que en los dientes a medir presentaran cavidades interproximales, microdoncia, anomalías de forma, fracturas o restauraciones que afectaran los contactos proximales.

Se procedió a realizar la medición de los dientes tanto de arcada superior como inferior, así como la longitud total de amabas arcadas con un calibrador digital de marca Surtek. Se recolectaron los datos en una hoja digital de Microsoft Excel en los que se anotaba el ancho mesiodistal de cada diente, la longitud de las arcadas, el sexo, la edad, la fecha de las impresiones.

Posteriormente se cálculo la discrepancia óseo dentaria de forma manual con una calculadora de marca Casio y se utilizó la aplicación iModelAnalysis2 para obtener la discrepancia óseo dental de cada uno de los modelos. Se realizó la estadística descriptiva y las pruebas de $t$ de Student en el programa SPSS versión 18.

\section{Resultados}

El promedio de edad de la muestra fue de 17 años, el 65\% fue de sexo femenino y el 35\% de sexo masculino. En el maxilar la media de la discrepancia ósea dentaria al calcularlo de forma manual fue de $-3.7 \mathrm{~mm}$, con una desviación estándar de $\pm 4.54 \mathrm{~mm}$. Al calcular la discrepancia con el programa iModelAnalysis 2 el promedio de la discrepancia óseo dentaria fue de $-3.22 \mathrm{~mm}$ con una desviación estándar de $\pm 4.62 \mathrm{~mm}$. El resto dela estadística descriptiva se encuentra en la tabla 1 .

\section{Tabla 1. Estadística descriptiva del maxilar}

\begin{tabular}{|c|c|c|}
\hline & iModelAnalisis2 & Manual \\
\hline Promedio & -2.77 & -3.32 \\
\hline Desviación estándar & 5.12 & 5.3 \\
\hline Máximo & 8.92 & 8.5 \\
\hline Mínimo & -15.66 & -16.3 \\
\hline
\end{tabular}

La discrepancia óseo dentaria calculada con la aplicación iModelAnalysis2 fue ligeramente menor que lo calculado de forma manual. Al comparar los resultados mediante la prueba de t de Student se encontró un valor de t de 1.18 , por lo que no se encontraron diferencias estadísticas significativas $(p<0.239)$.
Tabla 2. Estadística descriptiva de la mandíbula

\begin{tabular}{|c|c|c|}
\hline & iModelAnalisis2 & Manual \\
\hline Promedio & -3.22 & -3.70 \\
\hline Desviación estándar & 4.62 & 4.56 \\
\hline Máximo & 6.6 & 6.22 \\
\hline Mínimo & -11.5 & -12 \\
\hline
\end{tabular}

La estadística descriptiva de los resultados de la mandíbula se encuentran en la tabla 2. En el maxilar inferior la diferencia entre utilizar el programa y hacerlo de forma manual presentó una discrepancia de $0.5 \mathrm{~mm}$; Al comparar los resultados mediante la prueba de $\mathrm{t}$ de Student se encontró un valor de $t$ de 1.12 , por lo que no se encontraron diferencias estadísticas significativas $(\mathrm{p}<$ 0.262 ).

\section{Discusión}

El apiñamiento dental es consecuencia de una discrepancia del tamaño mesiodistal de los dientes y el volumen de las bases óseas que los sostienen, esta diferencia puede ser resultado de diversos factores, como: la forma de los dientes y las bases óseas, la longitud del hueso basal y el ancho transversal. ${ }^{10}$

Los análisis para el diagnóstico en ortodoncia deben tener en consideración que al utilizar normas obtenidas en otras poblaciones el tamaño de los dientes pueden variar por dimorfismo sexual, la influencia étnica y por el tipo de maloclusión. ${ }^{3,11,12}$ Existen varios ejemplos en los que se evalúan métodos (Moyers o Tanaka Johnston) en los que se predice el tamaño de los dientes que no han erupcionado con normas de diferentes grupos étnicos en los que el resultado no es el adecuado. ${ }^{13,-17}$

Con la aplicación iModelAnalysis2 se puede calcular el índice de Bolton, el perímetro del arco dental, el análisis de Carey's, el índice de Ashley-Howe's, el análisis de Pont, el índice de Linder Hart y el análisis de dentición mixta de Tanaka Johnston. La aplicación iModelAnalysis en su primera versión, fue evaluada la efectividad en el análisis de dentición mixta en la misma población de este estudio, los resultados de esa investigación no fueron positivos, ya que la aplicación no era efectiva en la predicción del tamaño mesiodistal de los dientes a erupcionar de una población de Nayarit, México. ${ }^{18}$

En este estudio se encontró que la aplicación iModelAnalysis 2 es efectiva para calcular la discrepancia óseo dentaria en el diagnóstico en ortodoncia, ya que utiliza únicamente los valores numéricos del mismo paciente para obtener 
la discrepancia óseo dentaria, y no en normas obtenidas en poblaciones diferentes a donde se aplique este método.

\section{Conclusiones}

1. El uso de la tecnología dentro del consultorio no esta peleado con los procedimientos que se realizan o utilizan rutinariamente para el correcto diagnóstico de cada paciente. Sin embargo, estas tecnologías que ahora tenemos a la mano, no siempre marcarán una diferencia y no necesariamente nos harán el trabajo menos pesado.

2. Cada profesional es libre de decisión el incorporar las nuevas tecnologías que van surgiendo con el día a día, o quedar un poco en lo cotidiano, y en ocasiones, más sencillos pasos a realizar de formas tradicionales.

3. Por lo tanto, realizar el cálculo de la discrepancia óseo dentaria de forma manual o utilizando la tecnología de un smartphone con la aplicación iModelanalisys 2 no alterará el diagnóstico que se le informará al paciente antes del inicio de su tratamiento.

\section{Referencias Bibliográficas}

1. Vellini F. Ortodoncia, diagnóstico y planificación clínica. Sao Paulo. Artes medicas: 2002; 143.

2. Bayona Y, Meneses A. Procedimientos clínicos para la corrección de la discrepancia de masa dentaria en pacientes con maloclusión clase I. Rev Estomatol Herediana. 2010; 20(1): 13-18.

3. Dos Santos R, Pithon M. Discrepancia dentaria de Bolton y finalización de ortodoncia: consideraciones clínicas. Int J Odontostomat. 2010. 4(1): 93-100.
4. Proffit W, Fields-Jr H, Sarver D. Ortodoncia contemporánea. 4ta edición. Barcelona. Elsevier Mosby: 2008; 195.

5. Andrade M, Aguilar E, BravoM. Análisis de Bolton en modelos de pacientes y relación con las diferentes maloclusiones. Revista Latinoamericana de Ortodoncia y Odontopediatría [Internet]. 2014[Citado 2016 febrero 11]: disponible en: https://www.ortodoncia.ws/publicaciones/2014/ art12.asp

6. Rakosi T, Jonas I. Atlas de ortopedia maxilar: diagnóstico. Barcelona. Ediciones Científicas y Técnicas S.A.: 1992; 222-4.

7. Morales L, Osorio E. Discrepancia óseo-dentaria severa. Tratamiento ortodóncico sin extracciones. Revista Gaceta Dental [Internet]. 2009 [Citado 2016 febrero 15]: disponible en: http:// www.gacetadental.com/2009/03/ discrepancia-seo-dentaria-severatratamiento-ortodncico-sin-extracciones-31297/\#

8. Ortiz J, Guzmán I. Corrección ortodóncico quirúrgica de clase III esquelética a través de avance y descenso del maxilar con injerto óseo. Revista Mexicana de Ortodoncia. 2013; 1 (1): 55-61.

9. Pavan K, Praveen K, Vasu S. Model analysis on a Smartphone. JCO. 2012. XLVI(6): 356-358.

10. Chávez Y, Saldívar O, Pérez H. Índice de Pont en modelos de estudio de pacientes con tratamiento ortodóncico terminado sin extracciones en la clínica de Ortodoncia de la DEPeI de la UNAM. Revista Mexicana de Ortodoncia. 2013; 1 (1): 7-12.
11. Carhuamaca G, Pérez L, Coronado M, Luque H. Estudio comparativo del tamaño mesiodistal entre dientes homólogos en dentición permanente. Odontol Sanmarquina. 2013; 16 (2):7-11.

12. Gutiérrez-Rojo JF, Reyes-Maldonado YS, Robles-Jiménez EA, Rojas-García AR. Comparación del tamaño mesiodistal de dientes permanentes con maloclusión de clase I, II, y III de Angle. Revista Española de Ortodoncia. 2013; 43 (4): 235-238.

13. Al-Bitar Z, Al-Omarib I, Sonbolc $\mathrm{H}$, Al-Ahmadd $\mathrm{H}$, Hamdane A. Mixed dentition analysis in a Jordanian population. Angle Orthod. 2008; 78 (4): 670-675.

14. Bernabe E, Flores-Mir C. Appraising number and clinical significance of regression equations to predict unerupted canines and premolars. Am J Orhtod Dentofacial Orthop. 2004; 126 (2): 228-230.

15. Gutiérrez J, Rojas A, Lemus C, Reyes Y. Efectividad del análisis de Tanaka Johnston en una población de Nayarit. Revista Oral. 2011; 12 (39): 795-798.

16. Jaroontham J, Godgray K. Mixed dentition space analysis in a Thai population. Eur J Orthod. 1998; 68 (1): 21-28.

17. Arslan S, Dildes N, Kama J, Genc C. Mixed dentition analysis in a Turkish population. World J Orthod. 2009: 10 (2): 135-140.

18. Lara I, Gutiérrez J, Guerrero M, Aguiar E. Evaluación de la efectividad del análisis de dentición mixta del programa iModelAnalysis para Smartphone en la población de Nayarit. Odonto pediatría actual. 2013; 7 (2): 24-26. 